\title{
Nivel de la capacidad para el emprendimiento en estudiantes de ingeniería y arquitectura de una universidad pública del sureste de México
}

\section{Capacity Level for Entrepreneurship in Engineering and Architecture Students of a Public University in Southeastern Mexico}

Nível de capacidade de empreendedorismo em estudantes de engenharia e arquitetura de uma universidade pública no sudeste do México

María del Carmen Sandoval Caraveo

Universidad Juárez Autónoma de Tabasco, México maria.sandoval@ujat.mx

http://orcid.org/0000-0002-5482-3032

Edith Georgina Surdez Pérez*

Universidad Juárez Autónoma de Tabasco, México edith.2109@hotmail.com http://orcid.org/0000-0001-8731-9273

Abraham Gerardo Pérez Sandoval

Universidad Juárez Autónoma de Tabasco, México

Icp_sandoval88@hotmail.com http://orcid.org/0000-0003-0657-3021

*Autor de correspondencia 


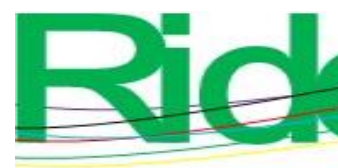
Revista Iberoamericana para la
Investigación y el Desarrollo Educativo
ISSN $2007-7467$

\section{Resumen}

Debido a que promueve la creación de empresas y del empleo, el emprendimiento es una actividad relacionada con el crecimiento económico. De ahí que en los últimos años las instituciones de educación superior hayan introducido esta temática dentro de sus programas educativos. El objetivo de esta investigación fue establecer la capacidad para el emprendimiento en estudiantes de ingeniería y arquitectura de una universidad pública del sureste de México. El método utilizado fue cuantitativo, de tipo descriptivo, con diseño no experimental transversal y alcance correlacional. La población participante fue de 3227 alumnos matriculados en los programas de ingeniería Mecánica Eléctrica, ingeniería Civil, ingeniería Eléctrica y Electrónica, ingeniería Química y Arquitectura. A partir de un muestreo aleatorio simple, la muestra fue de 343 estudiantes. Se aplicó al instrumento un análisis factorial confirmatorio a través del programa SPSS Amos, que comprobó un modelo sustentable de medición del constructo con tres factores: personal, recursos y social. El alfa de Cronbach reportó una confiabilidad de $\alpha=0.868$. Los resultados mostraron que $26 \%$ de los estudiantes considera no tener capacidad emprendedora y $23 \%$ poca facultad para ello. La estadística descriptiva reveló que las capacidades de emprendimiento de la muestra no son contundentes en ninguno de los factores analizados. La prueba post hoc de Bonferroni reveló diferencias significativas al comparar las capacidades emprendedoras entre los estudiantes de los programas educativos. Los participantes de ingeniería Mecánica Eléctrica mostraron mayor potencial para el emprendimiento. Asimismo, los alumnos de sexto y séptimo semestre y los que tienen 23 y 24 años poseen más capacidades de emprendimiento. No se encontraron diferencias significativas entre hombres y mujeres ni correlaciones importantes con la edad, el semestre y las calificaciones de los estudiantes. En conclusión, existe la necesidad de diseñar estrategias que coadyuven a robustecer las capacidades de emprendimiento en los sujetos de estudio por no encontrarse bien definidas.

Palabras clave: capacidad para el emprendimiento, empresa, ingeniería, universidad. 


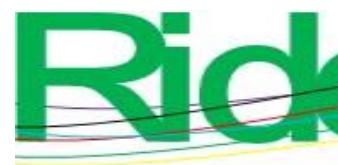

Revista Iberoamericana para la Investigación y el Desarrollo Educativo ISSN 2007-7467

\section{Abstract}

Because it has a key role in the creation of enterprises and jobs, entrepreneurship is an activity deeply related to economic growth. That is why, in the last couple of years, higher education institutions have introduced this subject in their academic programmes. The aim of this investigation was to establish the level of entrepreneurship in engineering and architecture students of a public university in Mexico. The method used was a quantitative approach of the descriptive type with a non-experimental, transversal and correlational design. The participating population consisted of 3227 students enrolled in either Electric Mechanic Engineering, Civil Engineering, Electric and Electronic Engineering, Chemical Engineering or Architecture. The sample of 343 students was obtained through a simple random sampling method. A factorial confirmatory analysis was applied to the instrument through the SPSS Amos program, which proved to be a sustainable model to measure a three-factor construct: personal, resources and social. The Cronbach's alpha test presented a reliability of $\alpha=0.868$. The results showed that $26 \%$ of the students consider no to have the ability to be entrepreneurs and that $23 \%$ have little ability. The descriptive statistic revealed that the abilities are not well defined in any of the analyzed factors. Bonferroni's post hoc analysis demonstrated the significant differences when comparing the abilities between the students of these academic programmes. Students of Electric Mechanic Engineering have more potential to be entrepreneurs, as well as students in sixth and seventh semester and those in the 23 to 24-year-old range. No significant differences were found among men and women nor do important correlation with age, semester and grades. Therefore, it is concluded that it is necessary to design strategies to contribute to strengthen the, not well determined, abilities to be entrepreneurs on the subjects of this study.

Keywords: entrepreneur, business, engineering, university. 


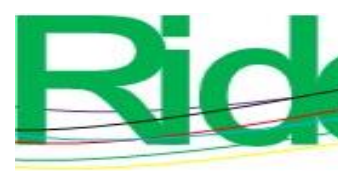
Revista Iberoamericana para la
Investigación y el Desarrollo Educativo
ISSN $2007-7467$

\section{Resumo}

Por promover a criação de negócios e empregos, o empreendedorismo é uma atividade relacionada ao crescimento econômico. Assim, nos últimos anos, as instituições de ensino superior têm introduzido esse tema em seus programas educacionais. O objetivo desta pesquisa foi estabelecer a capacidade de empreendedorismo em estudantes de engenharia e arquitetura de uma universidade pública do sudeste do México. O método utilizado foi quantitativo, descritivo, com delineamento transversal não experimental e escopo correlacional. A população participante foi de 3.227 alunos matriculados nos programas de Engenharia Elétrica Mecânica, Engenharia Civil, Engenharia Elétrica e Eletrônica, Engenharia Química e Arquitetura. A partir de uma amostragem aleatória simples, a amostra foi de 343 alunos. Uma análise fatorial confirmatória foi aplicada ao instrumento por meio do programa SPSS Amos, que verificou um modelo sustentável de mensuração do construto com três fatores: pessoal, recursos e social. $\mathrm{O}$ alfa de Cronbach relatou confiabilidade de $\alpha=$ 0,868. Os resultados mostraram que $26 \%$ dos alunos consideram não ter capacidade empreendedora e $23 \%$ pouco corpo docente para isso. A estatística descritiva revelou que as capacidades empreendedoras da amostra não são conclusivas em nenhum dos fatores analisados. O teste post hoc de Bonferroni revelou diferenças significativas ao comparar as habilidades empreendedoras entre alunos em programas educacionais. Os participantes da Engenharia Elétrica Mecânica apresentaram maior potencial de empreendedorismo. Da mesma forma, os alunos do sexto e sétimo semestres e os de 23 e 24 anos apresentam maior capacidade empreendedora. Não houve diferenças significativas entre homens e mulheres ou correlações significativas com a idade, semestre e notas dos alunos. Conclui-se que existe a necessidade de desenhar estratégias que ajudem a fortalecer as capacidades empreendedoras dos sujeitos do estudo por não serem bem definidas.

Palavras-chave: empreendedorismo, negócios, engenharia, universidade.

Fecha Recepción: Enero 2020

Fecha Aceptación: Agosto 2020 


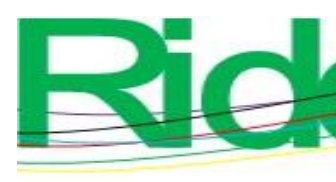

Revista Iberoamericana para la
Investigación y el Desarrollo Educativo
ISSN $2007-7467$

\section{Introducción}

El término emprendedor proviene del francés entrepreneur y surge a inicios del siglo XVI con objeto de nombrar a quienes estaban relacionados con el ámbito militar. En el siglo XVIII, los franceses aplicaron el concepto a los profesionales de la arquitectura y a quienes se dedicaban a construir puentes. En materia económica, el término fue definido por Richard Cantillon en 1755 como "el proceso de enfrentar la incertidumbre" (Hidalgo, 2014, p. 47). El vocablo inglés entrepreneurship es posible concebirlo como 'emprendimiento', 'empresarialidad', 'espíritu emprendedor' o 'empresariado' (Gutiérrez, 2011).

El emprendedor es un individuo que da inicio a una actividad económica, social o política. El emprendedor empresario "es una persona que identifica una oportunidad de negocio y organiza los recursos necesarios para ponerlo en marcha" (Hidalgo, 2014, p. 47). Desde la perspectiva económica, un empresario debe utilizar los elementos de producción de trabajo, conocimiento, tecnología, administración y capital para participar en actividades de producción. Las funciones de un empresario son diferentes a las de un empleado: este proporciona elementos de producción, aquel combina los elementos de producción para producir algo (Chen et al., 2013).

Al emprendedor es posible analizarlo a través de tres perspectivas. La primera comprende las teorías de motivación, de los rasgos y la cognitiva denominada psicológica; la segunda se refiere a la relación con los antecedentes familiares empresariales, y la tercera relaciona el emprendimiento con las actitudes, tiene que ver con el interés del individuo hacia la realización de actividades emprendedoras, lo que se considera como el factor principal del perfil emprendedor (Cabana, Cortés, Plaza, Castillo y Álvarez, 2013).

Sanabria, Morales y Ortiz (2015) mencionan que el emprendimiento es "la combinación entre la actitud y la capacidad de la persona que le permite llevar a cabo nuevos proyectos de cualquier índole, generalmente creativo" (p. 119). También ha sido descrito como un factor relacionado con el desarrollo de la economía de los países a través del fomento de empleos y del crecimiento de las empresas pequeñas. De allí que haya habido un incremento de investigaciones en el campo académico sobre este tema y un mayor interés por parte de las instituciones gubernamentales en promover actividades emprendedoras (Berríos, 2017). 


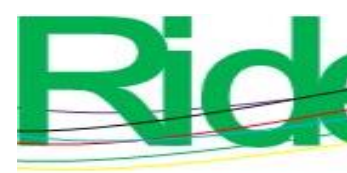

Revista Iberoamericana para la Investigación y el Desarrollo Educativo ISSN 2007 - 7467

Al respecto, Hatt (2018) menciona que de entre las conceptualizaciones de emprendimiento ninguna es suficiente por sí misma, ya que muchas personas con rasgos aparentemente empresariales no han establecido nuevas empresas y otras que han sido fundadoras de empresas no muestran un enfoque empresarial.

Para que surja una empresa, en primer lugar debe de haber una intención emprendedora, esto es, "el autoreconocimiento de la convicción de crear un negocio y la planificación consciente para su realización en un tiempo futuro" (Soria, Zuniga y Ruiz, 2016, p. 26). Los mismos Soria et al. (2016) señalan que los valores de un individuo, así como su ámbito cultural, familiar, social y educativo, influyen en su interés de crear una empresa.

En este sentido, Sánchez, Caggiano y Hernández (2011) opinan que las competencias emprendedoras se deben enseñar desde una edad temprana y hasta llegar al nivel universitario, considerando que las aptitudes y la personalidad del individuo representan factores importantes para el éxito y que la universidad, por su cercanía con el mercado laboral y por ser un espacio generador de conocimiento, se encuentra en un lugar importante para potenciar el emprendimiento en los estudiantes. Sánchez et al. (2011) agregan que la educación emprendedora, sumada a una actitud positiva de los jóvenes dirigida a la creación de empresas, propicia la incubación de nuevas formas empresariales, donde la ciencia y la tecnología son protagonistas.

Teniendo en cuenta todo lo anterior, los investigadores y la sociedad en general han incrementado en las últimas décadas su interés en comprender el fomento al emprendimiento y el papel de las universidades en el desarrollo económico y social (Guerrero et al., 2016). De tal modo que, acorde a la relevancia que tienen las instituciones de educación superior en el desarrollo social y económico de los países, han incluido en años recientes la temática del emprendimiento dentro de sus programas educativos y actividades para fomentar el espíritu emprendedor en los estudiantes (Cantillo, Piña, Gómez y Volpe, 2013; Sanabria et al., 2015).

Siguiendo a Núñez y Núñez (2016), la educación en la escuela debe dirigirse a fomentar el emprendimiento por el hecho de que los emprendedores contribuyen de manera importante al crecimiento económico, y puede hacerse incluyendo asignaturas que fomenten la educación emprendedora y estén dirigidas hacia la creación de empresas (fines económicos), o difundirla en los planes de estudios con el propósito de adoptar un enfoque interdisciplinario encaminado a la realización de proyectos sociales o productivos. 


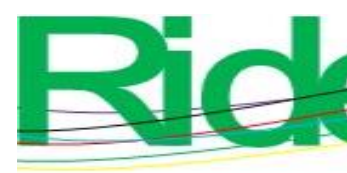

Revista Iberoamericana para la Investigación y el Desarrollo Educativo ISSN $2007-7467$

Así pues, las universidades deben ser apoyadas para que asuman un rol clave en promover el desarrollo emprendedor. El profesor, por su parte, debe prepararse para esta nueva forma de enseñanza que conlleva establecer acciones pedagógicas, verdaderos cambios en la práctica docente, que se traduzcan en una participación constante y en una capacitación dirigida a desarrollar las aptitudes relacionadas con el emprendimiento (Vera et al. 2008). Para el emprendimiento, según Chen et al. (2013), la educación tiene como objetivo cultivar la conciencia empresarial, el pensamiento y las habilidades a través de métodos educativos.

En este orden de ideas, la globalización demanda individuos emprendedores que respondan a las necesidades de nuevos conocimientos, donde las instituciones de educación superior juegan un papel preponderante en la educación para el emprendimiento tanto de circunstancias favorables como de riesgos implícitos en la actividad de emprender. Es así que la educación, además de promover el desarrollo de habilidades para la creación de empresas, debe tender hacia la motivación de cada individuo para fomentar acciones emprendedoras (Osorio y Pereira, 2011). Promover el espíritu empresarial y que la educación se dirija hacia el emprendimiento es una forma de aumentar el número de emprendedores para estimular el crecimiento económico (Rauch y Hulsink, 2015).

Existen estudios que han destacado la importancia de fomentar dicho espíritu emprendedor en las instituciones de educación superior, tal y como el de Cabana et al. (2013), quienes analizaron, a través de un estudio cuantitativo, las capacidades emprendedoras en alumnos de educación superior. Para la investigación en cuestión se consideraron cuatro factores: atributos del emprendedor, capacidades interpersonales, capacidades frente al riesgo y actitudes. La muestra se conformó por 389 alumnos de ocho licenciaturas adscritos a universidades privadas, institutos y centros de formación técnica. Los resultados muestran que los estudiantes poseen un nivel avanzado en las capacidades emprendedoras resultado del proceso de enseñanza-aprendizaje y de la cultura organizacional dirigida hacia el fomento del emprendimiento en los centros de educación superior. Cabana et al. (2013) concluyeron que los estudiantes tienen las condiciones para desarrollar e implementar oportunidades de negocios.

Otra investigación acerca de las capacidades emprendedoras en la educación superior es la de Rauch y Hulsink (2015), cuyo objetivo fue identificar si la educación para el emprendimiento tiene efectos en el comportamiento empresarial. Los hallazgos de este estudio de corte cuasiexperimental señalan que cuando existe participación de los 


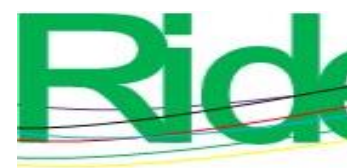
Revista Iberoamericana para la
Investigación y el Desarrollo Educativo
ISSN $2007-7467$

involucrados se presenta un aumento de las actitudes emprendedoras y se muestran mayores intenciones empresariales al terminar el programa. Y confirman que la educación para el emprendimiento se asocia a la creación de nuevos negocios.

Igualmente vale la pena mencionar el estudio elaborado por Alvarado y Rivera (2011) con 255 alumnos de las áreas económicas y administrativas en una facultad de Colombia. Este par de autores realizó una investigación exploratoria con enfoque mixto para identificar las características de emprendimiento en la población ya mencionada. Sus hallazgos apuntan a que tres cuartas partes de los estudiantes presentan interés para iniciar actividades empresariales. En términos de distribución de género, identificaron que hay un equilibro en esta propensión. Además, al descubrir que más de la mitad procede de familias con negocios propios, sugieren que el emprendimiento tiene una relación con el ambiente familiar. En el apartado de conclusiones, Alvarado y Rivera (2011) subrayan que la universidad debe potenciar las iniciativas emprendedoras de sus estudiantes a través de una estructura adecuada de sus planes y programas de estudio para contribuir al desarrollo económico de las regiones.

Los hallazgos encontrados en estos estudios evidencian que la universidad, a través de sus procesos de aprendizaje, tiene un destacado papel en el desarrollo de las capacidades emprendedoras y en la creación de empresas. Sin embargo, estos esfuerzos han sido realizadas en su mayoría con estudiantes de las áreas económicas y administrativas, por lo que existe, entonces, la necesidad y pertinencia de realizar pesquisas con alumnos que cursan otros programas educativos en el nivel superior para determinar diferencias y semejanzas y, en consecuencia, tener un panorama más amplio del comportamiento emprendedor en la población universitaria.

Por tales motivos, el objetivo de esta investigación fue establecer el nivel de la capacidad para el emprendimiento en estudiantes de ingeniería y arquitectura de una universidad pública del sureste de México. 


\section{Materiales y métodos}

\section{Participantes}

La población se integró por estudiantes de ingeniería y arquitectura de una universidad pública situada en el sureste mexicano. De una población total de 3227 alumnos, fueron encuestados 343. Los participantes fueron seleccionados a través de un muestreo aleatorio simple. El número de participantes por cada uno de los programas educativos se calculó de manera proporcional tomando como base la cantidad total de alumnos. Para aplicar el instrumento de investigación, el criterio de inserción fue que estudiaran del cuarto semestre en adelante, por considerar a estos estudiantes con más vivencias dentro de la universidad.

En el estudio participaron 81 estudiantes de ingeniería Mecánica Eléctrica, 84 de ingeniería Civil, 30 de ingeniería Eléctrica, 65 de ingeniería Química y 83 de Arquitectura. Las edades oscilaban entre los 19 y 26 años. De entre los 19 a 20, había 78 (22.7 \%); de entre $\operatorname{los} 21$ a 22, 156 (45.5\%); de entre $\operatorname{los} 23$ y 24, 88 (25.7\%), y de entre los 25 a 26 años, 21 $(6.1 \%)(\mathrm{M}=21.92, \mathrm{DT}=1.69)$. En términos de género y estado, $272(79.3 \%)$ eran hombres y $71(20.7 \%)$ mujeres; $312(91 \%)$ estaban solteros y 31 (9\%) casados. En relación con el semestre, $95(27.7 \%)$ cursaban el cuarto y quinto; 89 (25.9\%) el sexto y séptimo; 99 $(28.9 \%)$ el octavo y noveno, y $60(17.5 \%)$ el décimo y onceavo semestre. El promedio en las calificaciones tenía un rango de 7 a 10: 84 (24.5\%) tenían entre 7 y 7.9; otros 232 $(67.6 \%)$ contaban con un promedio de 8 a $8.9(67.6 \%)$ y $27(7.9 \%)$ resultaron con calificaciones entre 9 y $10(\mathrm{M}=8.19$, DT $=0.489)$.

\section{Instrumento}

Se recurrió a la adaptación realizada por Pérez (2019) del instrumento diseñado por Cabana et al. (2013) para medir las capacidades emprendedoras en estudiantes universitarios. Pérez (2019) realizó modificaciones en la redacción para que los ítems quedaran en escala tipo Likert y en sentido positivo. Esta versión se conformó de 22 ítems, con cinco opciones de respuesta: "Totalmente en desacuerdo" (0), "En desacuerdo" (1), "Ni de acuerdo ni en desacuerdo" (2), “De acuerdo" (3), “Totalmente de acuerdo" (4). El autor Pérez (2019) reportó una validez de constructo a través de un análisis factorial exploratorio con tres factores: personal, recursos y social. 
Para contextualizar la validez del instrumento a la población de los estudiantes de ingeniería y arquitectura reportada en este trabajo, se decidió aplicar un análisis factorial confirmatorio, que comprobó un modelo sustentable de medición. Esta prueba arrojó 18 ítems, las medidas de ajuste se reportan en la tabla 1.

Tabla 1. Pruebas de bondad de ajuste

\begin{tabular}{|l|l|l|}
\hline Medidas & Valor favorable & Valor obtenido \\
\hline $\begin{array}{l}\text { La razón de ji al cuadrado } \\
\text { sobre los grados de libertad }\end{array}$ & $<2.1$ y 3 & 2.43 \\
\hline Índice de bondad de ajuste & $\geq 0.90$ & 0.906 \\
\hline Índice de Tucker Lewis & $>0.90$ & 0.908 \\
\hline $\begin{array}{l}\text { Índice de ajuste } \\
\text { comparative }\end{array}$ & 0.90 & 0.921 \\
\hline $\begin{array}{l}\text { Error cuadrático medio de } \\
\text { aproximación }\end{array}$ & $<0.05$ a 0.08 & 0.060 \\
\hline
\end{tabular}

Fuente: Elaboración propia

La confiabilidad del instrumento se comprobó a través del alfa de Cronbach, que reportó valores aceptables para cada uno de los factores. El resultado se presenta en la tabla 2.

Tabla 2. Confiabilidad del instrumento

\begin{tabular}{|l|l|}
\hline Factor & Alfa de Cronbach \\
\hline Personal & 0.836 \\
\hline Recursos & 0.700 \\
\hline Social & 0.787 \\
\hline Confiabilidad total & 0.868 \\
\hline
\end{tabular}

Fuente: Elaboración propia

Las definiciones de los factores se muestran en la tabla 3. 


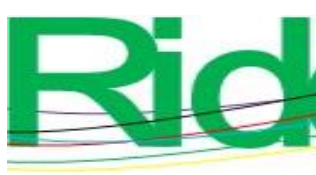

Revista Iberoamericana para la Investigación y el Desarrollo Educativo ISSN $2007-7467$

Tabla 3. Definición de los factores de la variable capacidades de emprendimiento

\begin{tabular}{|l|l|}
\hline Factor & Definición \\
\hline Personal & $\begin{array}{l}\text { Capacidades de los estudiantes para } \\
\text { concebir y hacer prosperar un negocio. }\end{array}$ \\
\hline Recursos & $\begin{array}{l}\text { Opinión o creencia del estudiante acerca de } \\
\text { los apoyos que le proporcionan los } \\
\text { profesores y la universidad para promover } \\
\text { el emprendimiento. }\end{array}$ \\
\hline Social & $\begin{array}{l}\text { Es la importancia que el estudiante le da a } \\
\text { las opiniones de sus compañeros con } \\
\text { relación a su determinación por emprender } \\
\text { un negocio. }\end{array}$ \\
\hline
\end{tabular}

Fuente: Elaboración propia con base en Pérez (2019)

\section{Procedimiento de recolección de datos}

La recolección de datos se realizó en la cafetería, en los salones de clases y en los laboratorios de la universidad. Se utilizó papel y lápiz. Los alumnos fueron informados de que no debían anotar su nombre en el cuestionario para conservar el anonimato y obtener información veraz. Los datos se analizaron a través de estadísticos descriptivos, inferenciales y de correlación con el paquete estadístico SPSS.

\section{Resultados}

En el análisis de frecuencias los datos mostraron una distribución normal con un valor mínimo de 0.72 y un valor máximo de 12 ; una curtosis de 1.706, una asimetría de -0.635 , una media de 8.27, una mediana de 8.31, una moda de 7.96 y una desviación estándar de 1.67 .

Las capacidades de emprendimiento se determinaron al clasificar el total de las respuestas en cuatro grupos. En el primer grupo estaban los estudiantes con puntuación menor o igual al percentil 25; en el segundo, quienes estaban encima del percentil 25 pero debajo del 50; en el tercer grupo, quienes se encontraban arriba del percentil 50 pero menor al 75, y en el cuarto grupo, los alumnos que se encontraban encima del percentil 75 (tabla 4). Acorde a los resultados, $26.1 \%$ de los estudiantes carecen de capacidades emprendedoras y 


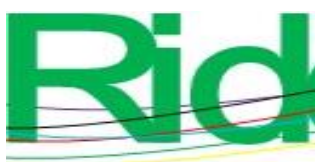

Revista Iberoamericana para la Investigación y el Desarrollo Educativo ISSN 2007 - 7467

$23.4 \%$ poseen muy pocas. Sin embargo, cabe resaltar que un cuarto de la población presenta alto potencial para el emprendimiento.

Tabla 4. Distribución de frecuencias de las capacidades emprendedoras de los estudiantes de ingeniería y arquitectura

\begin{tabular}{|l|c|c|c|}
\hline \multicolumn{1}{|c|}{\begin{tabular}{c}
\multicolumn{1}{|c|}{ Capacidades } \\
emprendedoras
\end{tabular}} & Percentil & Rango & \% \\
\hline $\begin{array}{l}\text { Los estudiantes no presentan } \\
\text { capacidad emprendedora. }\end{array}$ & 25 & $0.72-7.31$ & 26.1 \\
\hline $\begin{array}{l}\text { Los estudiantes presentan } \\
\text { poca capacidad } \\
\text { emprendedora. }\end{array}$ & 50 & $7.32-8.31$ & 23.4 \\
\hline $\begin{array}{l}\text { Los estudiantes presentan } \\
\text { capacidad emprendedora. }\end{array}$ & 75 & $8.32-9.41$ & 25.2 \\
\hline $\begin{array}{l}\text { Los estudiantes presentan alta } \\
\text { capacidad emprendedora. }\end{array}$ & 100 & $9.42-12$ & 25.3 \\
\hline
\end{tabular}

Fuente: Elaboración propia

Los resultados globales de las medidas de tendencia central señalaron que los puntajes se encuentran entre "Ni de acuerdo ni en desacuerdo" (2) y "De acuerdo" (3). Ningún factor alcanzó el valor que señalara estar de acuerdo de forma contundente. El factor social reportó la media más baja y la mayor desviación estándar (tabla 5).

Tabla 5. Descriptivos por factor y global de las capacidades emprendedoras

\begin{tabular}{|l|c|c|c|c|}
\hline Descriptivos & Factor personal & Factor recursos & Factor social & Global \\
\hline Media & 2.72 & 2.88 & 2.67 & 2.75 \\
\hline Mediana & 2.78 & 3.00 & 2.80 & 2.77 \\
\hline Moda & 3.00 & 3.00 & 2.60 & 2.65 \\
\hline D.E. & 0.64 & 0.68 & 0.76 & 0.55 \\
\hline N & 343 & 343 & 343 & 343 \\
\hline
\end{tabular}

Fuente: Elaboración propia 


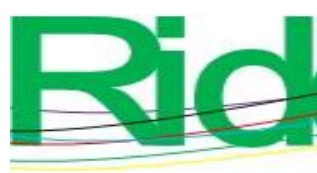

Revista Iberoamericana para la Investigación y el Desarrollo Educativo ISSN $2007-7467$

Por otra parte, se realizó el análisis de varianza (Anova) para identificar diferencias significativas por cada factor de las capacidades de emprendimiento entre los programas educativos (tabla 6).

Tabla 6. Comparación de las medias poblacionales de las capacidades emprendedoras por programa educativo

\begin{tabular}{|c|c|c|c|c|c|}
\hline Factores & Programa educativo & $\mathbf{N}$ & Media & $\mathbf{F}$ & Sig. \\
\hline \multirow[t]{7}{*}{ Personal } & & & & 1.774 & 0.134 \\
\hline & Mecánica Eléctrica & 81 & 2.86 & & \\
\hline & Civil & 84 & 2.62 & & \\
\hline & Eléctrica y Electrónica & 30 & 2.67 & & \\
\hline & Química & 65 & 2.76 & & \\
\hline & Arquitectura & 83 & 2.67 & & \\
\hline & Total & 343 & 2.72 & & \\
\hline \multirow[t]{7}{*}{ Recursos } & & & & 4.454 & $0.002 *$ \\
\hline & Mecánica Eléctrica & 81 & 3.10 & & \\
\hline & Civil & 84 & 2.73 & & \\
\hline & Eléctrica y Electrónica & 30 & 3.09 & & \\
\hline & Química & 65 & 2.85 & & \\
\hline & Arquitectura & 83 & 2.79 & & \\
\hline & Total & 343 & 2.88 & & \\
\hline \multirow[t]{7}{*}{ Social } & & & & 3.397 & $0.010^{*}$ \\
\hline & Mecánica Eléctrica & 81 & 2.92 & & \\
\hline & Civil & 84 & 2.59 & & \\
\hline & Eléctrica y Electrónica & 30 & 2.75 & & \\
\hline & Química & 65 & 2.57 & & \\
\hline & Arquitectura & 83 & 2.56 & & \\
\hline & Total & 343 & 2.67 & & \\
\hline
\end{tabular}

Nota: $* p \leq 0.05$

Fuente: Elaboración propia 

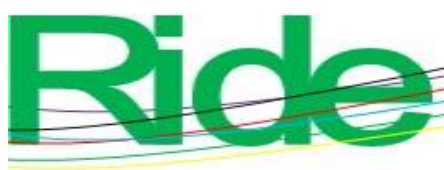

Como puede observarse en la tabla 6, existen diferencias estadísticamente significativas entre los programas educativos con los factores Recursos y Social. Para conocer con exactitud entre cuáles programas se encontraban estas diferencias, se aplicó la prueba post hoc de Bonferroni, que las identificó entre ingeniería Mecánica Eléctrica e ingeniería Civil (.004) y con Arquitectura (0.030) en el factor Recursos. El resultado de la media indica que los ingenieros Mecánicos perciben más capacidad para emprender un negocio. En el factor Social, se encontraron las diferencias entre ingeniería Mecánica Eléctrica con ingeniería Civil (.048), con ingeniería Química (.047) y con Arquitectura (.020); también aquí los ingenieros Mecánicos registraron mayor capacidad de emprendimiento. 


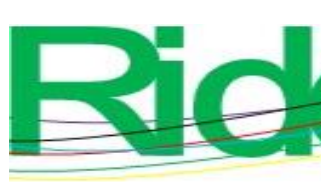

Revista Iberoamericana para la Investigación y el Desarrollo Educativo ISSN 2007 - 7467

Tabla 7. Comparación de las medias poblacionales de las capacidades emprendedoras por semestre

\begin{tabular}{|c|c|c|c|c|c|c|}
\hline Factores & Semestre & $\mathbf{N}$ & Media & D.E. & $\mathbf{F}$ & Sig. \\
\hline \multirow[t]{6}{*}{ Personal } & & & & & 5.913 & $0.001 *$ \\
\hline & $4 .^{\circ}$ y $5^{\circ}$ & 95 & 2.51 & 0.66 & & \\
\hline & $6 .^{\circ} \mathrm{y} 7 .^{\circ}$ & 89 & 2.89 & 0.61 & & \\
\hline & $8 .^{\circ}$ y $9 .^{\circ}$ & 99 & 2.72 & 0.64 & & \\
\hline & $10 .^{\circ} \mathrm{y} 11 .^{\circ}$ & 60 & 2.81 & 0.57 & & \\
\hline & Total & 343 & 2.72 & 0.64 & & \\
\hline \multirow[t]{6}{*}{ Recursos } & & & & & 1.032 & 0.379 \\
\hline & $4 .^{\circ}$ y $5 .^{\circ}$ & 95 & 2.81 & 0.70 & & \\
\hline & $6 .^{\circ} \mathrm{y} 7 .^{\circ}$ & 89 & 2.95 & 0.67 & & \\
\hline & $8 .^{\circ}$ y $9 .^{\circ}$ & 99 & 2.85 & 0.74 & & \\
\hline & $10 .^{\circ} \mathrm{y} 11 .^{\circ}$ & 60 & 2.96 & 0.58 & & \\
\hline & Total & 343 & 2.88 & 0.68 & & \\
\hline \multirow[t]{6}{*}{ Social } & & & & & 3.237 & $0.022 *$ \\
\hline & $4 .^{\circ}$ y $5 .^{\circ}$ & 95 & 2.58 & 0.59 & & \\
\hline & $6 .^{\circ} \mathrm{y} 7 .^{\circ}$ & 89 & 2.86 & 0.78 & & \\
\hline & $8 .^{\circ}$ y $9 .^{\circ}$ & 99 & 2.55 & 0.83 & & \\
\hline & $10 .^{\circ} \mathrm{y} 11 .^{\circ}$ & 60 & 2.72 & 0.82 & & \\
\hline & Total & 343 & 2.67 & 0.76 & & \\
\hline
\end{tabular}

Nota: ${ }^{*} p \leq 0.05$

Fuente: Elaboración propia

La tabla 7 permite ver que existen diferencias estadísticamente significativas entre las capacidades de emprendimiento de los alumnos con el semestre en los factores Personal y Social. La prueba post hoc de Bonferroni mostró de manera puntual que las diferencias se encontraban en el factor Personal entre los estudiantes que cursan cuarto y quinto semestre con los de sexto y séptimo (0.001) y con los de décimo y onceavo (0.029). Además, según los resultados de la media, quienes cursan sexto y séptimo poseen más capacidades de emprendimiento. En el factor Social, las diferencias se identificaron entre los estudiantes que cursan sexto y séptimo con los de octavo y noveno (030). Los resultados de la media muestran que los estudiantes de sexto y séptimo suelen tener mayor capacidad emprendedora. No se 


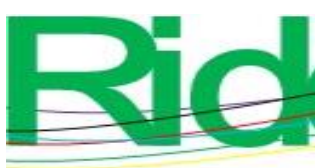

Revista Iberoamericana para la Investigación y el Desarrollo Educativo ISSN 2007 - 7467

encontraron diferencias estadísticamente significativas entre las capacidades de emprendimiento en relación con las calificaciones de los estudiantes.

Tabla 8. Comparación de las medias poblacionales de las capacidades emprendedoras por edad de los alumnos

\begin{tabular}{|c|c|c|c|c|c|c|}
\hline Factor & Edad & $\mathbf{N}$ & Media & D.E. & $\mathbf{F}$ & Sig. \\
\hline \multirow[t]{6}{*}{ Personal } & & & & & 2.853 & $0.037 *$ \\
\hline & 19 y 20 & 78 & 2.56 & 0.63 & & \\
\hline & 21 y 22 & 156 & 2.71 & 0.61 & & \\
\hline & 23 y 24 & 88 & 2.84 & 0.65 & & \\
\hline & 25 y 26 & 21 & 2.83 & 0.80 & & \\
\hline & Total & 343 & 2.72 & 0.64 & & \\
\hline \multirow[t]{6}{*}{ Recursos } & & & & & 2.686 & $0.047 *$ \\
\hline & 19 y 20 & 78 & 2.82 & 0.74 & & \\
\hline & 21 y 22 & 156 & 2.81 & 0.70 & & \\
\hline & 23 y 24 & 88 & 3.06 & 0.59 & & \\
\hline & 25 y 26 & 21 & 2.90 & 0.63 & & \\
\hline & Total & 343 & 2.88 & 0.68 & & \\
\hline \multirow[t]{6}{*}{ Social } & & & & & 3.13 & $0.026^{*}$ \\
\hline & 19 y 20 & 78 & 2.64 & 0.61 & & \\
\hline & 21 y 22 & 156 & 2.57 & 0.77 & & \\
\hline & 23 y 24 & 88 & 2.81 & 0.85 & & \\
\hline & 25 y 26 & 21 & 2.96 & 0.75 & & \\
\hline & Total & 343 & 2.67 & 0.76 & & \\
\hline
\end{tabular}

Nota: ${ }^{*} p \leq 0.05$

Fuente: Elaboración propia

En la tabla 8, por su parte, el Anova muestra diferencias significativas entre las capacidades emprendedoras con la edad de los estudiantes. Posteriormente, la prueba post hoc de Bonferroni indicó que las diferencias específicas estaban entre los estudiantes entre 19 y 20 con los de 23 y 24 años (0.032) en el factor Personal. En el factor Recursos, la 


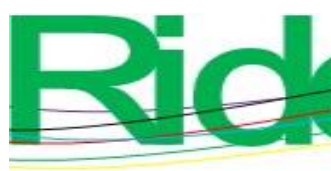

Revista Iberoamericana para la Investigación y el Desarrollo Educativo ISSN 2007 - 7467

diferencia se encontró entre los de 21 y 22 con los de 23 y 24 años (0.046). Esta prueba no arrojó diferencias en el factor Social.

Tabla 9. Correlaciones entre los factores de emprendimiento con el promedio en las calificaciones, la edad y el semestre

\begin{tabular}{|l|l|l|l|l|l|l|}
\hline Factores & Personal & Recursos & Social & Promedio & Edad & Semestre \\
\hline Personal & 1 & $0.548^{* *}$ & $0.450^{* *}$ & 0.036 & $0.149^{* *}$ & $.128^{*}$ \\
\hline Recursos & & 1 & $0.408^{* *}$ & 0.037 & $0.107 *$ & 0.055 \\
\hline Social & & & 1 & 0.104 & $0.123 *$ & 0.010 \\
\hline Promedio & & & 1 & $-0.130^{*}$ & -0.093 \\
\hline Edad & & & & 1 & $0.600^{* *}$ \\
\hline Semestre & & & & & 1 \\
\hline$* *$ La correlación es significativa en el nivel 0.01 (dos colas). & & \\
\hline$*$ La correlación es significativa en el nivel 0.05 (dos colas). & & \\
\hline
\end{tabular}

Fuente: Elaboración propia

El análisis de correlación de Pearson arrojó correlaciones positivas muy débiles entre los tres factores de las capacidades emprendedoras con la edad. En lo referente al semestre que estudiaban, se identificó una correlación positiva muy débil solo con el factor Personal; no hubo correlaciones con el promedio en las calificaciones (tabla 9).

\section{Discusión}

La presente investigación muestra las capacidades de emprendimiento a través de los factores denominados Personal, Recursos y Social en la población de estudiantes matriculados en la División Académica de Ingeniería y Arquitectura de una universidad pública del sureste mexicano. Los resultados señalaron que $26.1 \%$ de los estudiantes carecen de capacidades para emprender un negocio y $23.4 \%$ perciben poca facultad para ello, lo que devela un área de oportunidad para fomentar las actividades de emprendimiento en la población de estudio. Estos resultados difieren de lo reportado por Alvarado y Rivera (2011) y Cabana et al. (2013), quienes encontraron que los estudiantes de nivel superior se inclinan por las actividades relacionadas con el emprendimiento y muestran capacidades emprendedoras aceptables, las cuales son resultado de la enseñanza dirigida hacia esta actividad. 


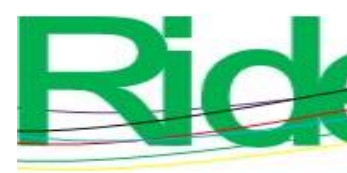

Revista Iberoamericana para la Investigación y el Desarrollo Educativo ISSN 2007 - 7467

De acuerdo con la escala Likert utilizada en el instrumento de investigación, ninguno de los factores indicó un valor que determinara a los estudiantes con capacidades de emprendimiento de manera contundente. Este resultado indica que las capacidades para emprender no se encuentran bien formadas en los estudiantes, en especial en el factor Social, que reportó la media más baja, aunque se haya encontrado mayor variabilidad en las respuestas en este factor.

En la comparación de las medias poblacionales, la prueba post hoc de Bonferroni reportó mayor capacidad de emprendimiento en el factor Recursos y en el Social en los estudiantes de ingeniería Mecánica; en el factor Recursos con Ingeniería Civil y Arquitectura; en el factor Social con ingeniería Civil, ingeniería Química y Arquitectura. Frente a esta información vale la pena reflexionar respecto al punto de vista de Hatt (2018), quien señala que, si bien la educación superior es un lugar adecuado para desarrollar el emprendimiento, existe una falta de consenso sobre la mejor manera de educar a los estudiantes con el objetivo de desarrollar su espíritu empresarial.

Otro hallazgo del estudio fueron las diferencias encontradas en el factor Personal y en el factor Social con el semestre, ya que los resultados de las medias indicaron que los de sexto y séptimo poseen mayores capacidades para emprender un negocio. Aquí se abre una compuerta no prevista: la posibilidad de un estudio posterior para identificar las causas de este resultado y generar propuestas con miras a fomentar el emprendimiento con mayor interés en alumnos que cursan otros semestres. Un dato interesante fue que las calificaciones de los estudiantes no influyen en la capacidad para emprender un negocio; se esperaría que los alumnos con calificaciones altas mostraran mayor tendencia hacia el emprendimiento, pero se encontró que los estudiantes poseen iguales habilidades para la creación de negocios en cuanto a calificaciones se refiera.

Respecto a la edad de los estudiantes, se determinó que quienes tienen entre 23 y 24 años poseen mayor capacidad de emprendimiento en los factores Personal y Recursos; sin embargo, en el factor Social, la capacidad para el emprendimiento es similar a pesar de la variabilidad en la edad. En lo concerniente al género, los datos indicaron igualdad de capacidades emprendedoras entre hombres y mujeres, lo cual concuerda con lo dicho por Alvarado y Rivera (2011), quienes también hablan de un equilibrio en este rubro.

En el análisis realizado para verificar la existencia de correlaciones, se confirmó que, a mayor promedio en las calificaciones, mayor edad y avance en los semestres, la capacidad 


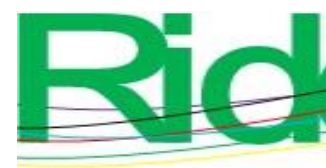

Revista Iberoamericana para la Investigación y el Desarrollo Educativo ISSN 2007-7467

emprendedora no se incrementa debido a que las correlaciones encontradas fueron muy débiles.

\section{Conclusiones}

Las universidades, al ser generadoras de conocimientos, tienen en sus manos la posibilidad de educar para el emprendimiento. Emprender conlleva la creación de empleos y, por ende, mejorar las condiciones de vida de la población. La investigación aquí presentada mostró los resultados del análisis de las capacidades emprendedoras en los estudiantes de ingeniería y arquitectura de una universidad pública.

Así, permitió determinar que la mitad de la población de los estudiantes no presenta capacidad para el emprendimiento. Derivado de los resultados, se reconoce la necesidad de diseñar estrategias que posibiliten potenciar las capacidades emprendedoras en los sujetos de estudio, debido a no encontrarse de forma contundente en los tres factores analizados. Asimismo, se requieren alternativas específicas para cada uno de los programas educativos en virtud de que cada uno tiene sus propias características.

Los datos obtenidos en la investigación proceden de un instrumento elaborado con rigor científico que puede ser aplicado en otros contextos similares. El presente trabajo abre la posibilidad de otros estudios de corte cualitativo para identificar las causas de los resultados aquí expuestos; en este sentido, considerando que aquí se reportó la media más baja de nivel de emprendimiento en el factor Social, se sugiere una línea de investigación que permita descubrir cómo se caracterizan los apoyos de familiares, profesores y amigos para iniciativas emprendedoras de estudiantes. En el factor Personal, una línea de investigación para explorar es la de las actitudes juveniles que pueden canalizarse hacia el emprendimiento. 


\section{Referencias}

Alvarado, O. y Rivera, W. F. (2011). Universidad y emprendimiento, aportes para la formación de profesionales emprendedores. Cuadernos de Administración, 27(45), 61-74. Recuperado de https://www.redalyc.org/pdf/2250/225019868005.pdf.

Berríos, J. E. (2017). Características emprendedoras: ¿Potenciales determinantes de la actividad emprendedora? Revista Internacional de Administración \& Finanzas, 10(6), 1-15. Recuperado de http://www.theibfr2.com/RePEc/ibf/riafin/riaf-v10n62017/RIAF-V10N6-2017-1.pdf.

Cabana, R., Cortés, I., Plaza, D., Castillo, M. y Álvarez, A. (2013). Análisis de las capacidades emprendedoras potenciales y efectivas en alumnos de Centros de Educación Superior. Journal of Technology Management \& Innovation, 8(1), 65-75. Recuperado de https://doi.org/10.4067/S0718-27242013000100007.

Cantillo, F., Piña O., Gómez, A. y Volpe, I. (2013). El efecto de la formación en emprendimiento sobre la construcción de una mentalidad emprendedora en estudiantes de Ingeniería Industrial en una universidad de la ciudad de Barranquilla. Sotavento M.B.A., (21), 50-61. Recuperado de https://revistas.uexternado.edu.co/index.php/sotavento/article/view/3436.

Chen, S., Hsiao, H., Chang, J., Chou, C., Chen, C. and Shen, C. (2013). Can the entrepreneurship course improve the entrepreneurial intentions of students? International Entrepreneurship and Management Journal, 11(3), 557-569. Retrieved from https://psycnet.apa.org/record/2015-33976-006-

Guerrero, M., Urbano, D., Ramos, A., Ruiz J., Neira, I. y Fernández, A. (2016). Observatorio de Emprendimiento Universitario en España. Edición 2015-2016. Madrid, España: Crue Universidades Españolas-RedEmprendia-CISE. Recuperado de https://www.crue.org/Documentos\%20compartidos/Publicaciones/Observatorio\%20 de\%20Emprendimiento\%20Universitario/20161201_Observatorio\%20de\%20Empr endimiento\%20Universitario_informe_web.pdf.

Gutiérrez, G. (2011). Educación emprendedora en la universidad: Educando para el futuro. Retos, Revista de Ciencias de la Administración y Economía, 2(1), 49-68. Recuperado de https://www.redalyc.org/pdf/5045/Resumenes/Resumen_504550952004_1.pdf. 


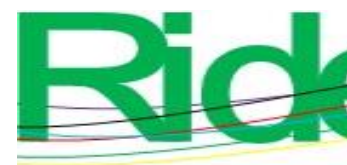
Revista Iberoamericana para la
Investigación y el Desarrollo Educativo
ISSN $2007-7467$

Hatt, L. (2018). Threshold concepts in entrepreneurship-the entrepreneurs' perspective. Education + Training, 60(2), 155-167. Retrieved from doi.org/10.1108/ET-08-20170119.

Hidalgo, L. F. (2014). La cultura del emprendimiento y su formación. Revista Alternativas, 15(1), 46-50. Recuperado de http://editorial.ucsg.edu.ec/ojsalternativas/index.php/alternativas-ucsg/article/view/8.

Núñez, L. y Núñez, M. (2016). Noción de emprendimiento para una formación escolar en competencia emprendedora. Revista Latina de Comunicación Social, (71), 10691089.

Osorio, F. y Pereira, F. (2011). Hacia un modelo de educación para el emprendimiento: una mirada desde la teoría social cognitiva. Cuadernos de Administración, 24(43), 13-33. Recuperado de http://www.redalyc.org/articulo.oa?id=20521435001.

Pérez, A. (2019). Emprendimiento y creación de empresas: Análisis de las capacidades emprendedoras en estudiantes universitarios de las ciencias económicas y administrativas. (tesis doctoral). Instituto Universitario Puebla, Villahermosa.

Rauch, A. and Hulsink. W. (2015). Putting Entrepreneurship Education Where the Intention to Act Lies: An Investigation into the Impact of Entrepreneurship Education on Entrepreneurial Behavior. Academy of Management Learning \& Education, 14(2), 187-204.

Sanabria, P. E., Morales, M. E. y Ortiz, C. (2015). Interacción universidad y entorno: marco para el emprendimiento. Educación y Educadores, 18(1), 111-134.

Sánchez, J., Caggiano, V. y Hernández, B. (2011). Competencias emprendedoras en la educación universitaria. International Journal of Developmental and Educational $\begin{array}{llll}\text { Psychology. } & \text { 1(3), } & \text { 19-28. }\end{array}$ http://www.redalyc.org/pdf/3498/349832330001.pdf.

Soria, K., Zuniga S. y Ruiz, S. (2016). Educación e intención emprendedora en estudiantes universitarios: un caso de estudio. Formación universitaria, 9(1), 25-34. Recuperado de https://scielo.conicyt.cl/pdf/formuniv/v9n1/art04.pdf.

Vera, P., Baquedano C., Ferrám, Y., Olavarría, S., Parra, E. y De Souza, B. (2008). Una innovación pedagógica para la formación de universitarios emprendedores. Revista da FAE, 11(2), 113-126. 


\begin{tabular}{|c|c|}
\hline Rol de Contribución & Autor (es) \\
\hline Conceptualización & $\begin{array}{l}\text { María del Carmen Sandoval Caraveo (igual) Edith Georgina } \\
\text { Surdez Pérez } \quad \text { (igual) Abraham Gerardo Pérez Sandoval } \\
\text { (igual) }\end{array}$ \\
\hline Metodología & $\begin{array}{l}\text { María del Carmen Sandoval Caraveo (igual) Edith Georgina } \\
\text { Surdez Pérez (igual) Abraham Gerardo Pérez Sandoval } \\
\text { (igual) }\end{array}$ \\
\hline Software & No aplica \\
\hline Validación & $\begin{array}{l}\text { María del Carmen Sandoval Caraveo (igual) Edith Georgina } \\
\text { Surdez Pérez (igual) Abraham Gerardo Pérez Sandoval } \\
\text { (igual) }\end{array}$ \\
\hline Análisis Formal & $\begin{array}{l}\text { María del Carmen Sandoval Caraveo (que apoya) Edith } \\
\text { Georgina Surdez Pérez (principal) Abraham Gerardo Pérez } \\
\text { Sandoval (que apoya) }\end{array}$ \\
\hline Investigación & $\begin{array}{l}\text { María del Carmen Sandoval Caraveo (igual) Edith Georgina } \\
\text { Surdez Pérez (igual) Abraham Gerardo Pérez Sandoval } \\
\text { (igual) }\end{array}$ \\
\hline Recursos & $\begin{array}{l}\text { María del Carmen Sandoval Caraveo (igual) Edith Georgina } \\
\text { Surdez Pérez (igual) Abraham Gerardo Pérez Sandoval } \\
\text { (igual) }\end{array}$ \\
\hline Curación de datos & $\begin{array}{l}\text { María del Carmen Sandoval Caraveo (igual) Edith Georgina } \\
\text { Surdez Pérez (igual) Abraham Gerardo Pérez Sandoval } \\
\text { (igual) }\end{array}$ \\
\hline $\begin{array}{l}\text { Escritura - Preparación del } \\
\text { borrador original }\end{array}$ & $\begin{array}{l}\text { María del Carmen Sandoval Caraveo (igual) Edith Georgina } \\
\text { Surdez Pérez } \quad \text { (igual) Abraham Gerardo Pérez Sandoval } \\
\text { (igual) }\end{array}$ \\
\hline $\begin{array}{l}\text { Escritura - Revisión y } \\
\text { edición }\end{array}$ & $\begin{array}{l}\text { María del Carmen Sandoval Caraveo (igual) Edith Georgina } \\
\text { Surdez Pérez (igual) Abraham Gerardo Pérez Sandoval } \\
\text { (igual) }\end{array}$ \\
\hline Visualización & $\begin{array}{l}\text { María del Carmen Sandoval Caraveo (igual) Edith Georgina } \\
\text { Surdez Pérez (igual) Abraham Gerardo Pérez Sandoval } \\
\text { (igual) }\end{array}$ \\
\hline Supervisión & $\begin{array}{l}\text { María del Carmen Sandoval Caraveo (principal) Edith } \\
\text { Georgina Surdez Pérez (que apoya) Abraham Gerardo Pérez } \\
\text { Sandoval (que apoya) }\end{array}$ \\
\hline $\begin{array}{l}\text { Administración de } \\
\text { Proyectos }\end{array}$ & $\begin{array}{l}\text { María del Carmen Sandoval Caraveo (principal) Edith } \\
\text { Georgina Surdez Pérez (que apoya) Abraham Gerardo Pérez }\end{array}$ \\
\hline
\end{tabular}




\begin{tabular}{|l|}
\hline \\
\hline \\
Investigación y el Desarrollo Educativo \\
ISSN $2007-7467$
\end{tabular}

